Volume 70, Number 1, Pages 468 482(2021)

DOI: $10.31801 /$ cfsuasmas. 644071

ISSN 1303-5991 E-ISSN 2618-6470

Received by the editors: November 7, 2019; Accepted: March 16, 2021

\title{
MEDICAL MODEL ESTIMATION WITH PARTICLE SWARM OPTIMIZATION
}

\author{
Murat SARI ${ }^{1}$, Arshed A. AHMAD ${ }^{2}$, and Hande USLU ${ }^{1}$ \\ ${ }^{1}$ Department of Mathematics, Yildiz Technical University, Istanbul 34220, TURKEY \\ ${ }^{2}$ Department of Mathematics, Yildiz Technical University, Istanbul 34220, TURKEY \\ ${ }^{2}$ Department of Computer, College of Education for Pure Science, Diyala University, IRAQ
}

\begin{abstract}
In this paper, a nonlinear medical model based on observational variables has been produced and the particle swarm optimization (PSO) technique, which is an effective technique to predict optimum parameters of the biomedical model, has been used. This study has been conducted on a dataset consisting of 539 subjects. For comparison purposes, nonlinear regression analysis, nonlinear deep learning, and nonlinear regression neural network methods are also considered and the PSO results appear to be slightly better than that of other methods. Built on observational variables and findings, the model is expected to be a good guide for healthcare professionals in diagnosing pathologies and planning treatment programs for their patients. It is therefore strongly believed that the article will be particularly useful for those interested in emerging biomedical models in various medical modelling areas such as infectious and hematological diseases such as anemia.
\end{abstract}

\section{INTRODUCTION}

The progress of mathematical models considered to produce medical outputs is a growing field in medical science. Mathematical models used for various reasons are important equipment to deal with the behaviour of a phenomenon. As underlined in the literature $[1,2,3,3,4,5]$, mathematical modelling has been achieved to be an essentially important tool for the analysis of pathological characteristics. The current medical model describes the relationship between the biomedical variables and the anemia types. Other important classes of medical models directly involve nonlinear models in the fundamental operation, and this generally means that one is considering the presence of nonlinear terms in medical models. Thus, the nature

2020 Mathematics Subject Classification. Primary 62P10, 65C20; Secondary 65K10.

Keywords and phrases. Anemia, medical modelling, nonlinear model, particle swarm optimization.

凶sarim@yildiz.edu.tr; arshed980@gmail.com-Corresponding author; usluh@yildiz.edu.tr

(D) 0000-0003-0508-2917; 0000-0003-1393-1253; 0000-0002-1642-1120. 
of nonlinear models is very important in many medical fields. The observational data are modelled by a function which is a nonlinear combination of the model parameters. Here the parameters are estimated for each of the variables in the nonlinear medical model, and this is the optimal model for more accurate prediction of the anemia types through the biomedical information.

Many models have been produced in dealing with different medical problems in the literature such as breast and ovarian cancers 6, colon and colorectal cancer [7, congenital heart disease 8, diabetic nephropathy [9], and osteoporosis 10]. A medical problem of frequently encountered is a set of data, which can be defined and described as a mathematical model having a set of parameters used to characterize the model. In this study, the main emphasis will be on the fitting parameters of the nonlinear medical model, which will be supposed to have some medical or mathematical significance through estimating best values in the set of the parameters. Therefore, the major aim here is to improve a nonlinear medical model to study the effect of the biomedical variables on the anemia types through a large number of the blood variables because there has been an increase in the incidence of anemia among various segments of society. As illustrated in the literature 11, 12, 13, 14, 15, the anemia of chronic disease, also called the anemia of chronic inflammation, was initially thought to be primarily associated with the infectious, inflammatory diseases. Some other estimation approaches $16,17,18,19$ were worked to analyze anemia problems while one of them considered metastatic tumours problem. Heuristic algorithms can be used effectively to find the optimal parameters of the nonlinear model in many medical studies. Therefore, the PSO is one of the most effective optimization algorithms that are applied for a wide set of complex optimization problems. In computational science, the PSO is a computational method that is used for solving a problem by having a population of candidate solutions. Therefore, these candidate solutions are created in a frequently used method to increase the likelihood that they are actual solutions.

Kennedy and Earhart 20 proposed the PSO approach to solve different problems in the literature $21,22,23,24,25,26,27,28,29]$, to estimate the parameters of nonlinear models. Besides, different mathematical method strategies are implemented to predict and optimize problems using the PSO with other methods $30,31,32,33,34$.

Despite the recognized advantages of conventional methods, most of them have different disadvantages such as difficulty in use, time-consuming, and high cost. In this case, optimization can be considered as an excellent competitor to the related methods. Different heuristic approaches have been improved in the recent two decades to simplify solving optimization problems, such as the PSO approach. The PSO has many advantages including simplicity of the implementation and rapid convergence, which are the most attractive features of this algorithm. It is reliable, robust, and in general, is an effective algorithm to improve meta-heuristic approaches $35,36,37,38,39$. In this study, the PSO has been proposed to predict 
the best parameter values of the nonlinear medical model. This algorithm is a typical tool for its ability to improve the complex search spaces, so, the PSO is popular in the academic community and that encouraged us to use it in dealing with the current medical problem.

The remaining contents are organized as follows. The samples used, the PSO algorithm, the predicted parameters and testing model are all considered in Section 2. The results of the estimated parameters, discussion and analysis have been given in Sections 3 and 4. Finally, conclusions and future research have been reported.

\section{Data And Methods}

2.1. Study samples. Anemia is a decrease in the blood susceptibility to transfer a sufficient amount of oxygen to the various tissues of the body because of the low level of haemoglobin in red blood cells or a decrease in the total number of red blood cells in the circulatory system, on this basis, tissue does not get enough oxygen to grow and sustain its various functions. Anemia is the most common one among the blood diseases in the world and one of the important clinical signs of underlying disorders 40 .

The data used for this study were collected from observations of blood variables and included 539 subjects provided from the literature 2, 41. Observations include individuals of the ages between (6-56) years old and 211 healthy subjects and 328 patients with anemia. Here we have samples for people and for each person readings of blood variables are 11, 12, 13, 40]: Hemoglobin (HB), Red Blood Cells (RBC), Mean Corpuscular Hemoglobin (MCH), White Blood Cell (WBC), Mean Corpuscular Volume (MCV), Hematocrit (HCT), Mean Corpuscular Hemoglobin Concentration (MCHC), Platelets (PLT), sex and age and some diseases of blood (Iron deficiency anemia (1), Deficiency Vitamin B12 (2), Thalassemia (3), Sickle cell (4) and Spherocytosis (5)). The anemia types and blood variables for our data are displayed in Table 1

TABle 1. Some samples from the data

\begin{tabular}{ccccccccccc}
\hline \hline HB & RBC & MCH & WBC & MCV & HCT & MCHC & PLT & Sex & Age & Anemia type \\
\hline 11.2 & 3.91 & 28.6 & 7.9 & 93 & 36.5 & 30.6 & 232 & 1 & 6 & 0 \\
17.5 & 5.55 & 31.6 & 14.1 & 92 & 50.9 & 34.5 & 318 & 2 & 23 & 0 \\
11.1 & 4.38 & 25.3 & 5.8 & 81 & 35.6 & 31.1 & 227 & 1 & 11 & 1 \\
3.84 & 10.5 & 27.3 & 6.1 & 91 & 35.1 & 29.9 & 538 & 2 & 38 & 1 \\
7.4 & 2.44 & 30.5 & 2.1 & 99 & 24.3 & 30.7 & 23 & 1 & 16 & 2 \\
2.42 & 7 & 28.9 & 54.3 & 97 & 23.4 & 29.9 & 307 & 2 & 28 & 2 \\
10.8 & 4.33 & 25 & 9.5 & 81 & 35.3 & 30.7 & 287 & 2 & 30 & 3 \\
7.3 & 3.08 & 23.7 & 5.8 & 82 & 25.2 & 28.9 & 199 & 1 & 53 & 3 \\
6.9 & 2.75 & 25.2 & 4.4 & 81 & 22.4 & 30.9 & 420 & 2 & 16 & 4 \\
2.66 & 8.2 & 30.8 & 15.7 & 90.6 & 24.1 & 34 & 437 & 1 & 29 & 4 \\
8.4 & 3.14 & 26.9 & 65.4 & 82 & 25.6 & 32.9 & 220 & 1 & 9 & 5 \\
7.3 & 4.11 & 17.8 & 5.6 & 64 & 26.3 & 27.9 & 482 & 2 & 16 & 5 \\
\hline
\end{tabular}


2.2. Modelling. Nonlinear models are important tools because life is nonlinear and many physical processes encountered in the physical environment such as biology, medicine, chemistry, physics, and other areas are better represented by a nonlinear model. Therefore, most processes are governed by nonlinear models in various fields of science such as the estimation of the parameters of a nonlinear medical model for predicting the anemia types.

A nonlinear model can be given in a basic form,

$$
y=f(x, b)+\epsilon,
$$

where $y, x, b, f()$ and $\epsilon$ indicate the observations, the vector of the coefficients, the known nonlinear function and the unobserved random variable that adds noise to the nonlinear relationship, respectively.

A nonlinear medical model describes the relationship between the dependent and independent variables when the behaviour of the model is nonlinear. Many nonlinear models can be proposed for dealing with anemia problem. In this regard, here, several attempts were made to obtain the best results, depending on the biomedical variables and the exponent of each input variable of interest. Other types of nonlinear models for the data were also considered and it was noted that the current model was the best among these models to obtain accurate results. In addition, those powers of the variables in the model have been investigated to find the optimum parameter values of the model, so as to obtain the best fitting for the data. It is well-known that the model order is chosen according to the number of bends needed on the structure of interest. Each increase in the exponent produces one more bend in the fitted structure. Therefore, it is tried to be found the multivariable nonlinear function that best fits the specific structure in the data.

The derived model is hence as follows:

$$
\hat{\mathbf{y}}=\frac{b_{0}}{E_{1}+E_{2}}+\epsilon,
$$

Here the denominator of Equation (2) is separated into two parts as $E_{1}, E_{2}$. Thus the separated parts are clearly expressed as:

$E_{1}=b_{1}(H B)^{6}+b_{2}(R B C)^{5}+b_{3}(M C H)^{4}+b_{4}(W B C)^{3}+b_{5}(S e x)^{2}$

$E_{2}=b_{6}(H C T)+b_{7}(M C H C)^{\frac{1}{2}}+b_{8}(P L T)^{\frac{1}{3}}+b_{9}(M C V)^{\frac{1}{4}}+b_{10}(A g e)$

where $\hat{\mathbf{y}}$ is the types of anemia, $b_{i}, 0 \leq i \leq 10$, are the parameters to be determined. Here HB, RBC, MCH, WBC, MCV, HCT, MCHC, PLT indicate Hemoglobin, Red Blood Cell, Mean Corpuscular Hemoglobin, White Blood Cell, Mean Corpuscular Volume, Haematocrit, Mean Corpuscular Hemoglobin Concentration, Platelets, respectively.

2.3. Particle Swarm Optimization. The PSO [20, is a population-based stochastic approach for solving continuous and discrete optimization problems. The method optimizes a problem by iteratively trying for an improved solution concerning a given measure of quality. They inspired from the behavior of birds and fish. 
These animals have a major role in the development of the algorithm to escape from dangerous situations or to search for food by looking at each other.

In the PSO, the particles move in the search space and randomly selected particles search an improved solution. Therefore, the position of a particle represents a candidate solution for the current optimization problem. All particles look for better positions in the search space at the end of each iteration by changing their speed according to the rules inspired by behavioral models of bird flocking. Because of each iteration, the position and velocity vectors are expressed as follows:

$$
\begin{gathered}
V_{i}^{(t+1)}=\omega V_{i}^{t}+c_{1} r_{1}\left(P_{\text {best }}-X_{i}{ }^{t}\right)+c_{2} r_{2}\left(G_{\text {best }}-X_{i}{ }^{t}\right) \\
X_{i}{ }^{(t+1)}=X_{i}{ }^{t}+V_{i}{ }^{(t+1)}
\end{gathered}
$$

where $t, \omega, c_{1}, c_{2}, r_{1}, r_{2}, X_{i}{ }^{t}, V_{i}{ }^{t}, P_{\text {best }}$ and $G_{b e s t}$, indicate iteration number, weight parameter (inertia weight), cognitive parameter, social parameter, random numbers in the interval $(0,1)$, position of individual $i$ at iteration $t$, velocity of individual $i$ at iteration $t$, the best local value of each particle, the best value of swarm, respectively. Here $t$ represents the current iteration, $r_{1}, r_{2}$ are the random numbers uniformly distributed between 0 and 1 , acceleration coefficients $c_{1}, c_{2}$ are usually between 0 and 4 . $\omega$ denotes the inertia weight and usually decreases from around 0.9 to around 0.4 during the computation.

The particles keep the best value in their memory. This value, $P_{b e s t}$, is listed for each particle and the best value is selected according to the fitness function such as $G_{\text {best }} 39,42,43$.

Parameter selection of the PSO has an important influence on the performance and efficiency of the algorithm. Determining the optimal parameters to achieve optimum performance is a complex issue. There is no general method for determining the optimum parameters or the stopping condition for iterations, in fact the parameters are chosen according to user experience as stated in the literature 44 45. In this paper, the PSO is used to estimate the parameters of the nonlinear medical model for deriving an accurate model. The process is done by finding a rapid convergence in fewer iterations and provides an accurate estimation of the parameters of the nonlinear medical model. (see Tables 233 )

2.4. Test for the model. Determination of the coefficient is a measure showing the rate of the contribution of the independent variables in the interpretation of the change in the dependent variable and to know that the model explains the change in the relationship between all blood variables, sex, and age and the anemia types. Then it is given as follow:

$$
R^{2}=1-\frac{S S E}{S S T}
$$


The model estimation is selected with the confidence interval of $95 \%$ and adjusted (Type III) sum of square tests. The computation formulae can be given as follows:

$$
S S T=\sum_{j=1}^{n}\left(y_{j}-\bar{y}\right)^{2},
$$

and

$$
S S E=\sum_{j=1}^{n}\left(y_{j}-\hat{y}_{j}\right)^{2}=\sum_{j=1}^{n} e_{j}^{2} .
$$

Here $S S T, S S E, y_{j}, \bar{y}, \hat{y}_{j}, e_{j}$ and $n$ represent the sum of squared total, the sum of squared error, the observations, mean value of the observations, the value estimated, the residual and the number of observations, respectively.

The model uses the mean square residuals to measure the extent of the propagation of the random error around the nonlinear model. The MSE indicate the concentration of data around the line. The smaller MSE gives more accurate the results 46, 47. Then it is given by

$$
M S E=\frac{1}{n} \sum_{j=1}^{n} e_{j}^{2} .
$$

2.5. Estimation of the parameters of the nonlinear medical model. The nonlinear function $f$ in Equation (1) has parameters given by $b=\left(b_{1}, b_{2}, \ldots\right)$. This study aims at estimating the parameters $b$ obtained by minimizing the sum of the squared error function $S S E(b)$ under the consideration of the PSO.

Hence, the cost (fitness) function in the PSO is selected as $S S E(b)$, specifically:

$$
S S E(b)=\sum_{i=1}^{n}\left(y_{i}-f\left(x_{i}, b\right)\right)^{2} .
$$

For instance, for the model in Equation (2),

$$
S S E(b)=\sum_{i=1}^{539}\left(y_{i}-\frac{b_{0}}{E_{1}+E_{2}}\right)^{2},
$$

$E_{1}=b_{1}(H B)^{6}+b_{2}(R B C)^{5}+b_{3}(M C H)^{4}+b_{4}(W B C)^{3}+b_{5}(S e x)^{2}$, $E_{2}=b_{6}(H C T)+b_{7}(M C H C)^{\frac{1}{2}}+b_{8}(P L T)^{\frac{1}{3}}+b_{9}(M C V)^{\frac{1}{4}}+b_{10}($ Age $)$.

Here $y_{i}$ represents dependent observations, $b_{i}, 0 \leq i \leq 10$, are the parameters to be determined.

The main parameters of the PSO method are $\omega, c_{1}, c_{2}$, and the size of the swarm. The settings for these parameters are decided according to how to optimize the search space. The inertia weight is used to control the effect of the previous velocities on the current velocity. Thus, the parameter $\omega$ regulates the trade-off between 
the global and local exploration capabilities of the swarm and also provides a balance between the global and local exploration capacity of the swarm and to find a better solution [21,30,48.

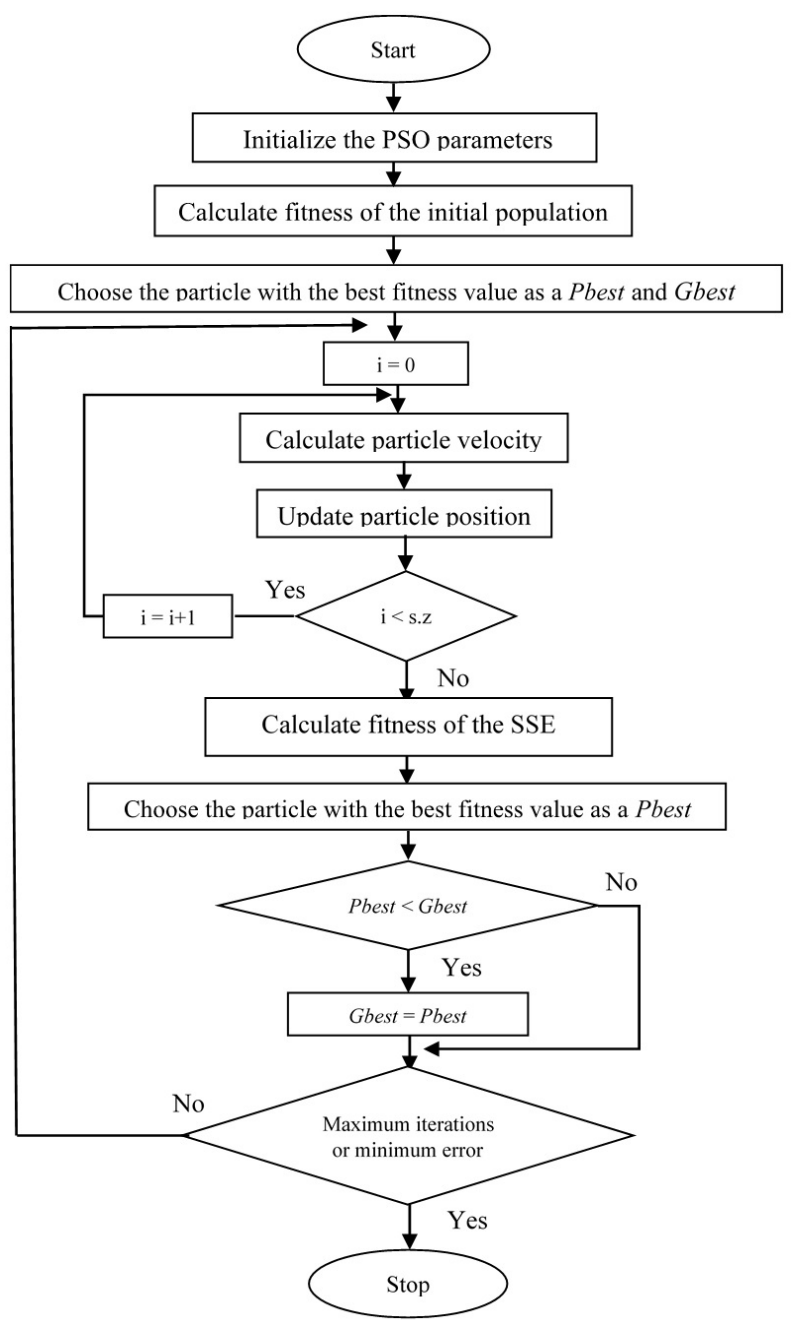

Figure 1. The PSO algorithm

The usual choices for acceleration coefficients are cognitive parameter $c_{1}$ and social parameter $c_{2}$, usually, $c_{1}$ is equal to $c_{2}$ ranged between 0 and 4 . The swarm size plays a very important role in the PSO, as is the complexity and sturdiness 
of the algorithm. As shown in Figure 1 the PSO algorithm was inspired by the literature 21, 42 .

\section{The Results For the estimated Parameters of the MOdel}

The current study concentrates on obtaining the best estimation of parameters via the PSO for the currently derived nonlinear medical model to discover the effect of blood variables, sex, and age on the types of anemia. Thus, the parameters of the nonlinear medical model are estimated through the PSO algorithm (see Table 2), and the produced results for various versions of the models through the minimum error are illustrated in Table 3 . In the estimation step, when the number of iteration increases, the error is decreasing as seen in Figures 2.4. Notice that the iteration reaches its optimum level at 3000 . It is important to note that, nonlinear regression analysis, the nonlinear deep learning (LSTM) and nonlinear regression neural network methods have also been applied to compare our model results. The results of the currently derived model are more accurate than the other competitors (see Table 4).

TABLE 2. Estimation of the parameters of the nonlinear medical model by the PSO algorithm

\begin{tabular}{ll|cccc}
\hline \hline Iteration Number & & 100 & 500 & 1000 & 3000 \\
\hline MSE & & 2.071 & 0.717 & 0.510 & 0.503 \\
\hline SST & & 1157.243 & 1157.243 & 1157.243 & 1157.243 \\
\hline SSE(b) & & 1116.277 & 386.518 & 275.358 & 271.148 \\
\hline$R^{2}$ & & 0.036 & 0.666 & 0.762 & 0.766 \\
\hline Constant & $b_{0}$ & -283.184 & -211.634 & -439.808 & -337.966 \\
HB & $b_{1}$ & -0.001 & -0.0001 & -0.0001 & -0.0002 \\
RBC & $b_{2}$ & -0.025 & -0.0001 & 0.029 & -0.002 \\
MCH & $b_{3}$ & 0.0001 & 0.0001 & 0.0003 & -0.00009 \\
WBC & $b_{4}$ & -1.177 & 0.0001 & 0.000006 & 0.00002 \\
MCV & $b_{5}$ & -9.257 & -1.456 & 1.006 & -2.600 \\
HCT & $b_{6}$ & 0.0001 & -0.895 & -7.688 & -8.733 \\
MCHC & $b_{7}$ & -10.285 & 1.720 & -72.156 & -40.975 \\
PLT & $b_{8}$ & -2.359 & -0.673 & -9.352 & -3.851 \\
Sex & $b_{9}$ & -4.206 & 1.551 & -115.177 & -191.734 \\
Age & $b_{10}$ & -2.024 & -0.909 & 0.159 & -0.256 \\
\hline
\end{tabular}

\section{Discussion and Analysis}

Previous researchers have considered a very limited number of blood variables or a few types of anemia $49,50,51$ to investigate various diseases, and they kept the 


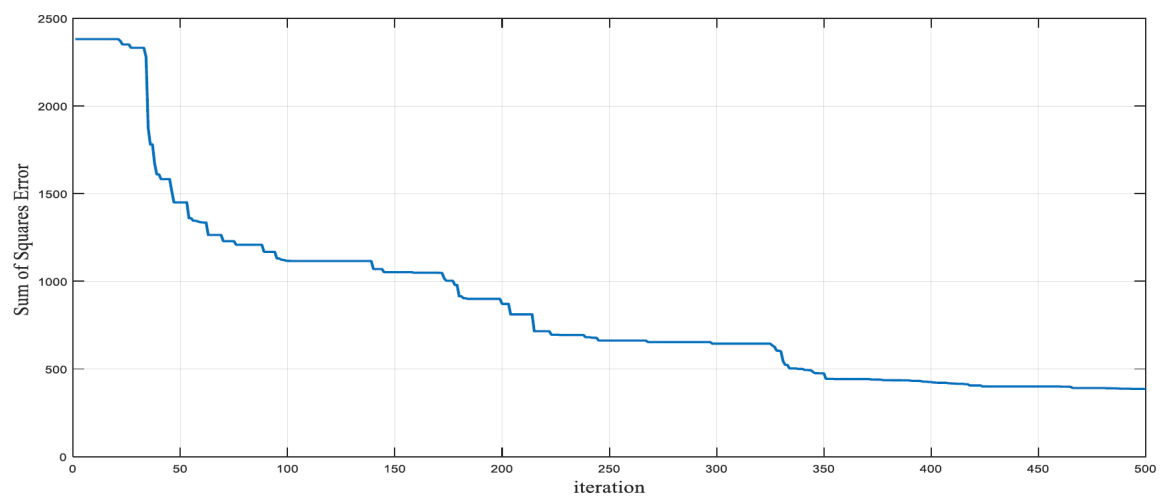

FiguRE 2. Behaviour of the sum of squared errors by the PSO when the iteration is 500

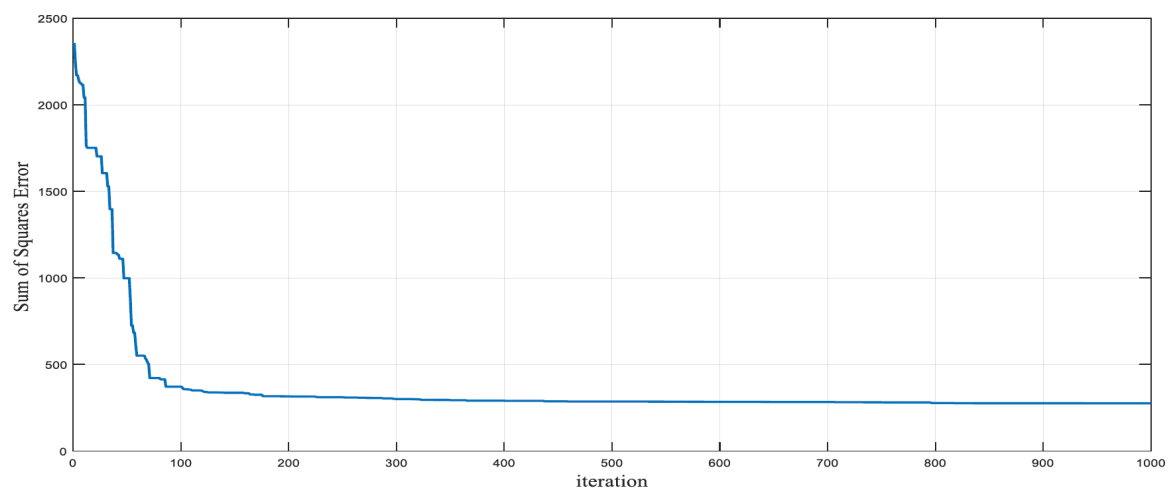

Figure 3. Behaviour of the sum of squared errors by the PSO when the iteration is 1000

number of observational variables and the anemia types in their studies very modest as opposed to the current study. Therefore, here, we focus on an optimum nonlinear medical model investigating the relationship between many blood variables and the types of anemia.

As opposed to the PSO approach, classical ways in dealing with the nonlinear model have some disadvantages as seen in the previous works $30,31,32,33,34$ with required a lot of cumbersome operations like matrix operations, gradient operations,

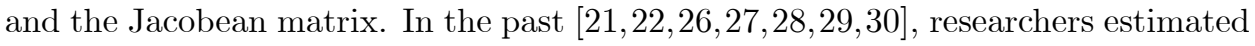
the parameters of a large number of various models by using the PSO. In the corresponding literature, they discussed various models/problems by using their 


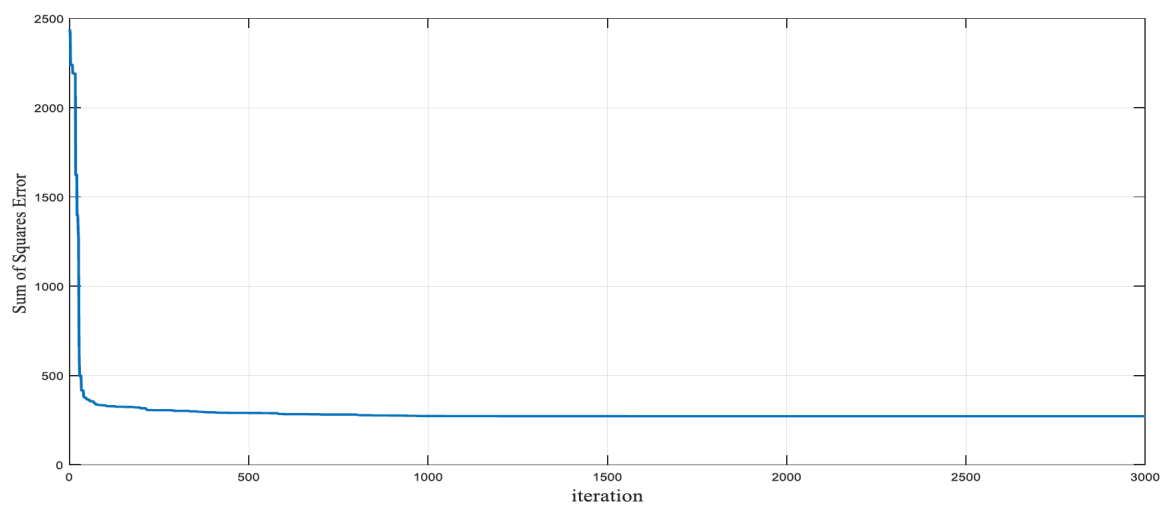

Figure 4. Behaviour of the sum of squared errors by the PSO when the iteration is 3000

TABLE 3. Estimation of the parameters of the various forms of the nonlinear medical model by the PSO algorithm

\begin{tabular}{llll}
\hline Models & SSE & $R^{2}$ & MSE \\
\hline Model 1 for (HB, sex and age) & 519.588 & 0.551 & 0.971 \\
Model 2 for (RBC, sex and age) & 940.242 & 0.188 & 1.744 \\
Model 3 for (MCH, sex and age) & 889.922 & 0.231 & 1.650 \\
Model 4 for (WBC, sex and age) & 939.826 & 0.188 & 1.757 \\
Model 5 for (MCV, sex and age) & 908.792 & 0.215 & 1.699 \\
Model 6 for (HCT, sex and age) & 734.005 & 0.366 & 1.372 \\
Model 7 for (MCHC, sex and age) & 907.781 & 0.216 & 1.697 \\
Model 8 for (PLT, sex and age) & 930.375 & 0.196 & 1.739 \\
Model 9 for (HB, RBC, sex and age) & 514.533 & 0.555 & 0.964 \\
Model 10 for (MCH, WBC, sex and age) & 882.851 & 0.237 & 1.638 \\
Model 11 for (MCV, HCT, sex and age) & 728.407 & 0.371 & 1.364 \\
Model 12 for (MCHC, PLT, sex and age) & 855.350 & 0.261 & 1.602 \\
Model 13 for (WBC, MCV, HCT, MCHC, sex and age) & 716.885 & 0.381 & 1.348 \\
Model 14 for (HB, RBC, MCH, PLT, sex and age) & 380.217 & 0.671 & 0.715 \\
\hline
\end{tabular}

own approaches. We have here studied a nonlinear model for a large amount of medical data on anemia to estimate the parameters of the model through the PSO.

In this work, the swarm size is calculated according to the structure of the medical model, the number of estimated parameters, and the search space $(-1000$ and 10). The algorithm parameters $c_{1}$ and $c_{2}$ have been selected as 1 and 3 , respectively. The termination criterion has been defined as the iteration limit. Specifically, the algorithm has been set to stop after different independent experiments for different iterations to verify the robustness of the estimation strategy. 
TABLE 4. Comparison of the results of the PSO with the three methods

\begin{tabular}{llll}
\hline Methods & SSE & MSE & $R^{2}$ \\
\hline PSO & 271.148 & 0.503 & 0.766 \\
Nonlinear regression analysis [[2] & 271.148 & 0.514 & 0.766 \\
Nonlinear Deep Learning Methods (LSTM) $[[2]$ & 273.465 & 0.560 & 0.760 \\
Nonlinear Regression Neural Networks $[2]$ & 287.826 & 0.534 & 0.752 \\
\hline
\end{tabular}

LSTM: Long Short Term Memory

Estimating the parameters of the medical model for improvement is a complicated task for classical algorithms. $b$ values refer to the estimated parameter values for the real parameters that the PSO obtains after randomly specifying the initial parameters of the model from the search space. After making different independent attempts and different iterations, 100, 500, 1000, and 3000 were taken to get the best parameters, and we achieved that goal at 3000 iterations (see Table 2 and Figures 2, 4).

Since the PSO algorithm is inherently random, the convergence behavior and the final predicted parameter values can be of interest. For the nonlinear model, the behavior of the error function is explained by the PSO approach which consists of the values estimated during the minimization process (see Figures 2 4). If Figures 2.4 are examined closely, the superiority variation to estimation accuracy for the parameter values of the medical model, $S S E=271.149, M S E=0.503$, and $R^{2}=$ 0.766 by the PSO, may be seen. This is important because the SSE and the MSE measure how well the data fit the model and anemia types, and concentration of data around the model line, which means a better fit for the model with the data. The model has been seen to be significantly effective on the prediction of anemia types, and the model explains $76.60 \%$ of the change in the relationship between the observational variables and the anemia types.

From the results obtained in Tables 2 and 3 , we see through the SSE, MSE and $R^{2}$ by using the PSO that the models produced in terms of a larger number of blood variables show a better correlation than the models produced in terms of fewer blood variables for predicting anemia types at the iteration of 3000 .

This study addresses the anemia prediction issue by the PSO compared to other methods including nonlinear regression analysis, the nonlinear deep learning method (LSTM), and the nonlinear regression neural network. The computed results showed that the PSO has the best fit to the initial dataset compared to the others (see Table 4 ).

\section{Conclusions and Future Research}

In this article, we have developed an alternative for the parameter estimation approach that relies on the PSO algorithm in the nonlinear medical model. As 
opposed to the PSO approach, classical methods have some disadvantages because they require many intricate mathematical operations. It can be concluded that the PSO algorithm has been seen to be an effective and very suitable parameter estimation method for the current nonlinear medical model. In addition, it should be noted that the PSO algorithm shows a tendency for rapid convergence; specifically, the algorithm converges for the model with the knowledge that the number of parameters is eleven. Therefore, the used parameter values are the latest and best results for securing a more realistic approach. Note that in the current problem the PSO approach has been found to be more accurate than both the nonlinear deep learning method (LSTM) and the nonlinear regression neural network. It can be accepted that the use of a relatively small amount of data or input variables could have seriously weakened our results and observations. For future research, various computational methods should be considered to estimate the parameters of the nonlinear medical model with this amount of data.

Authors Contribution Statement All authors conceived of the presented idea. Arshed A.A., developed the study, performed the computations. Hande U., verified the methods and and investigated results. Murat S., supervised and edited all the findings of this work. Arshed A.A. and Hande U., designed the draft manuscript. All authors discussed the results and contributed to the final manuscript.

Declaration of Competing Interests The authors declare that they have no known financial interests or personal relationships that conflict with each other affecting the study reported in this article.

Acknowledgement The authors would like to thank the anonymous reviewers for their valuable comments and suggestions for improving the paper and they would also like to thank kindly the editor of the journal.

\section{REFERENCES}

[1] Gulbandilar, E., Cimbiz, A., Sari, M., Ozden, H., Relationship between skin resistance level and static balance in type II diabetic subjects, Diabetes Research and Clinical Practice, 82(3) (2008), 335-339 https://doi.org/10.1016/j.diabres.2008.09.011

[2] Ahmad, A.A., Sari, M., Anemia prediction with multiple regression support in system medicinal internet of things, Journal of Medical Imaging and Health Informatics, 10(1) (2020) 261-267. https://doi.org/10.1166/jmihi.2020.2839

[3] Sari, M., Tuna, C., Prediction of pathological subjects using genetic algorithms, Computational and Mathematical Methods in Medicine, 2018, (2018). https://doi.org/10.1155/ 2018/6154025

[4] Sari, M., Cetiner, B.G., Predicting effect of physical factors on tibial motion using artificial neural networks, Expert Systems with Applications, 36(6) (2009), 9743-9746 https://doi. org/10.1016/j.eswa.2009.02.030

[5] Cetiner, B.G., Sari, M., Tibial rotation assessment using artificial neural networks, Mathematical and Computational Applications, 15(1) (2010), 34-44. https://doi.org/10.3390/ mca15010034 
[6] Botesteanu, D.A., Lipkowitz, S., Lee, J.M., Levy, D., Mathematical models of breast and ovarian cancers, Wiley Interdisciplinary Reviews: Systems Biology and Medicine, 8(4) (2016), 337-362. https://doi.org/10.1002/wsbm.1343

[7] Gilli, Q., Mustapha, K., Frayret, J.M., Lahrichi, N., Karimi, E., Patient model for colon and colorectal cancer care trajectory simulation, Health Science Journal, 11(6) (2017), 1-16. https://doi.org/10.21767/1791-809X.1000536

[8] Loke, Y.H., Harahsheh, A.S., Krieger, A., Olivieri, L.J., Usage of 3D models of tetralogy of Fallot for medical education: impact on learning congenital heart disease, BMC Medical Education, 17(1) (2017), 54. https://doi.org/10.1186/s12909-017-0889-0

[9] Soler, M.J., Riera, M., Batlle, D., New experimental models of diabetic nephropathy in mice models of type 2 diabetes: efforts to replicate human nephropathy, Experimental Diabetes Research, 2012, (2012). https://doi.org/10.1155/2012/616313

[10] Stephens, M.H., Grey, A., Fernandez, J., Kalluru, R., Faasse, K., Horne, A., Petrie, K.J., 3-D bone models to improve treatment initiation among patients with osteoporosis: A randomised controlled pilot trial,Psychology \&3 Health, 31(4) (2016), 487-497. https://doi.org/10.1080/ 08870446.2015 .1112389

[11] Rivera, S., Ganz, T., Animal models of anemia of inflammation, In Seminars in Hematology, 46(4) (2009), 351-357.https://doi.org/10.1053/j.seminhematol.2009.06.003

[12] Weiss, G., Goodnough, L.T., Anemia of chronic disease, New England Journal of Medicine, 352(10) (2005), 1011-1023. https://doi.org/10.1056/NEJMra041809

[13] Weiss, G., Gasche, C., Pathogenesis and treatment of anemia in inflammatory bowel disease, Haematologica, 95(2) (2010), 175-178. https://doi.org/10.3324/haematol.2009.017046

[14] Li, X., Dao, M., Lykotrafitis, G., Karniadakis, G.E., Biomechanics and biorheology of red blood cells in sickle cell anemia, Journal of Biomechanics, 50 (2017), 34-41. https://doi. org/10.1016/j.jbiomech.2016.11.022

[15] Kim, A., Rivera, S., Shprung, D., Limbrick, D., Gabayan, V., Nemeth, E., Ganz, T., Mouse models of anemia of cancer, PLoS One, 9(3) (2014), e93283. https://doi.org/10.1371/ journal.pone.0093283

[16] Berzuini, C., Franzone, P.C., Stefanelli, M., Viganotti, C., Iron kinetics: modelling and parameter estimation in normal and anemic states, Computers and Biomedical Research, 11(3) (1978), 209-227. https://doi.org/10.1016/0010-4809(78)90008-3

[17] Barosi, G., Cazzola, M., Morandi, S., Stefanelli, M., Perugini, S., Estimation of ferrokinetic parameters by a mathematical model in patients with primary acquired sideroblastic anaemia, British Journal of Haematology, 39(3) (1978), 409-423. https://doi.org/10.1111/ j.1365-2141.1978.tb01112.x

[18] Dey, S., Raheem, E., Multilevel multinomial logistic regression model for identifying factors associated with anemia in children 6-59 months in northeastern states of India, Cogent Mathematics, 3(1)(2016), 1159798. https://doi.org/10.1080/23311835.2016.1159798

[19] Mehrara, E., Forssell-Aronsson, E., Johanson, V., Kölby, L., Hultborn, R., Bernhardt, P., A new method to estimate parameters of the growth model for metastatic tumours, Theoretical Biology and Medical Modelling, 10(31) (2013), 1-12. https://doi.org/10.1186/ 1742-4682-10-31

[20] Eberhart, R., Kennedy, J., A new optimizer using particle swarm theory, In MHS'95. Proceedings of the Sixth International Symposium on Micro Machine and Human Science,IEEE, (1995), 39-43. https://doi.org/10.1109/MHS.1995.494215

[21] Ozsoy, V.S., Orkcu, H.H., Estimating the parameters of nonlinear regression models through particle swarm optimization, Gazi University Journal of Science, 29(1) (2016), 187-199.

[22] Abdullah, A., Deris, S., Mohamad, M.S., Anwar, S., An improved swarm optimization for parameter estimation and biological model selection, PLoS One, 8(4) (2013), e61258. https: //doi.org/10.1371/journal.pone.0061258 
[23] Chen, S., Yang, R., Yang, R., Yang, L., Yang, X., Xu, C., Xu, B., Zhang, H., Lu, Y., Liu, W., A parameter estimation method for nonlinear systems based on improved boundary chicken swarm optimization, Discrete Dynamics in Nature and Society, 2016, (2016). https: //doi.org/10.1155/2016/3795961

[24] Hong, X., Ding, Y., Ren, L., Chen, L., Huang, B., A weighted heteroscedastic Gaussian Process Modelling via particle swarm optimization, Chemometrics and Intelligent Laboratory Systems, 172 (2017), 129-38. https://doi.org/10.1016/j.chemolab.2017.11.019

[25] Chang, W.D., Cheng, J.P., Hsu, M.C., Tsai, L.C., Parameter identification of nonlinear systems using a particle swarm optimization approach, In2012 Third International Conference on Networking and Computing,, IEEE, (2012), 113-117. https://doi.org/10.1109/ICNC. 2012.24

[26] Chu, H.J., Chang, L.C., Applying particle swarm optimization to parameter estimation of the nonlinear Muskingum model, Journal of Hydrologic Engineering, 14(9) (2009), 1024-1027. https://doi.org/10.1061/(ASCE)HE.1943-5584.0000070

[27] Hosseini, M., Naeini, S.A., Dehghani, A.A., Khaledian, Y., Estimation of soil mechanical resistance parameter by using particle swarm optimization, genetic algorithm and multiple regression methods, Soil and Tillage Research, 157 (2016), 32-42.https://doi.org/10.1016/ j.still.2015.11.004

[28] Jau, Y.M., Su, K.L., Wu, C.J., Jeng, J.T., Modified quantum-behaved particle swarm optimization for parameters estimation of generalized nonlinear multi-regressions model based on Choquet integral with outliers, Applied Mathematics and Computation, 221 (2013), 282-295. https://doi.org/10.1016/j.amc.2013.06.050

[29] Jahandideh, H., Namvar, M., Use of PSO in Parameter Estimation of Robot Dynamics; Part One: No Need for Parameterization, In System Theory, Control and Computing, ICSTCC, 2012, 16th International Conference, IEEE, (2012), 1-6,.

[30] Erdogmus, P., Ekiz, S., Nonlinear Regression using Particle Swarm Optimization and Genetic Algorithm, International Journal of Computer Applications, 153(6) (2016). https://doi. org/10.5120/ijca2016912081

[31] Alfiyatin, A.N., Febrita, R.E., Taufiq, H., Mahmudy, W.F., Modeling house price prediction using regression analysis and particle swarm optimization, International Journal of Advanced Computer Science and Applications, 8 (2017). https://doi.org/10.14569/IJACSA. 2017.081042

[32] Samareh, H., Khoshrou, S.H., Shahriar, K., Ebadzadeh, M.M., Eslami, M., Optimization of a nonlinear model for predicting the ground vibration using the combinational particle swarm optimization-genetic algorithm, Journal of African Earth Sciences, 133 (2017), 36-45. https://doi.org/10.1016/j.jafrearsci.2017.04.029

[33] Satapathy, S.C., Murthy, J.V., Reddy, P.P., Misra, B.B., Dash, P.K., Panda, G., Particle swarm optimized multiple regression linear model for data classification, Applied Soft Computing, 9(2) (2009), 470-476. https://doi.org/10.1016/j.asoc.2008.05.007

[34] Sari, M., Tuna, C., Akogul, S., Prediction of tibial rotation pathologies using particle swarm optimization and K-means algorithms, Journal of Clinical Medicine, 7(4) (2018), 65. https: //doi.org/10.3390/jcm7040065

[35] Shi, Y., Eberhart, R., A modified particle swarm optimizer, In Evolutionary Computation Proceedings, 1998. IEEE World Congress on Computational Intelligence, The 1998 IEEE International Conference,, IEEE, (1998), 69-73. https://doi.org/10.1109/ICEC.1998.699146

[36] Kennedy, J., Eberhart, R., Particle swarm optimization, In Neural Networks, Proceedings, IEEE International Conference, 4 (1995), 1942-1948. http://doi.org/10.1109/ICNN.1995. 488968

[37] Yang, C., Simon, D., A new particle swarm optimization technique, 8th International Conference on Systems Engineering (ICSEng'05) (2005), 164-169. https://doi.org/10.1109/ ICSENG.2005.9 
[38] Marini, F., Walczak, B., Particle swarm optimization (PSO). A tutorial, Chemometrics and Intelligent Laboratory Systems, 149 (2015), 153-165.https://doi.org/10.1016/j.chemolab . 2015.08.020

[39] Poli, R., Kennedy J., Blackwell, T., Particle swarm optimization, Swarm Intelligence, 1(1) (2007), 33-57. http://doi.org/10.1007/s11721-007-0002-0

[40] Hoque, M., Hoque, E., Kader, S.B., Risk factors for anaemia in pregnancy in rural KwaZuluNatal, South Africa: implication for health education and health promotion, South African Family Practice, 51(1) (2009), 68-72. https://doi.org/10.1080/20786204.2009.10873811

[41] Sari, M., Ahmad, A.A., Anemia modelling using the multiple regression analysis, International Journal of Analysis and Applications 17(5) (2019), 838-849. https://doi.org/10. 28924/2291-8639-17-2019-838

[42] Alzaidi, K.M., Bayat, O., Uçan, O.N., A heuristic approach for optimal planning and operation of distribution systems, Journal of Optimization, 2018, (2018). https://doi.org/10. $1155 / 2018 / 6258350$

[43] Bai, Q., Analysis of particle swarm optimization algorithm, Computer and Information Science, 3(1) (2010), 180. https://doi.org/10.5539/cis.v3n1p180

[44] He, Y., Ma, W., Zhang, J., The Parameters Selection of PSO Algorithm influencing on performance of Fault Diagnosis, MATEC Web of Conferences, 63, 02019 (2016). https: //doi.org/10.1051/matecconf/20166302019

[45] Wang, Y., Li, B., Yin, L., Wu, J., Wu, S., Liu, C.,Velocity-controlled particle swarm optimization (PSO) and its application to the optimization of transverse flux induction heating apparatus, Energies, 12(487) (2019).https://doi.org/10.3390/en12030487

[46] Seber, G.A., Wild, C.J., Nonlinear regression, John Wiley \\& Sons, Hoboken, NJ. 2003.

[47] Rudolf, F.J., William, J.W., Ping, S., Regression analysis: statistical modeling of a response variable, Elsevier, USA, 2006.

[48] Mohanty, S.D., Particle swarm optimization and regression analysis-I, Astronomical Review, 7(2) (2012), 29-35. https://doi.org/10.1080/21672857.2012.11519700

[49] Ngwira, A., Kazembe, L.N., Analysis of severity of childhood anemia in Malawi: a Bayesian ordered categories model, Open Access Medical Statistics, 6 (2016), 9-20. https://doi.org/ 10.2147/OAMS.S95159

[50] Chen, Y.M., Miaou, S.G., A kalman filtering and nonlinear penalty regression approach for noninvasive anemia detection with palpebral conjunctiva images, Journal of Healthcare Engineering, (2017). https://doi.org/10.1155/2017/9580385

[51] Habyarimana, F., Zewotir, T., Ramroop, S., Structured additive quantile regression for assessing the determinants of childhood anemia in Rwanda, International Journal of Environmental Research and Public Health, 14(6) (2017), 652 https://doi.org/10.3390/ijerph14060652 